\title{
djb-Mitgliederversammlung am 26. September 2013 in Leipzig: Kandidaturen für den Bundesvorstand
}

Am 28. September 2013 findet im Rahmen des 40. Bundeskongresses in Leipzig die Mitgliederversammlung des djb statt. Der gesamte Bundesvorstand wird satzungsgemäß neu gewählt. Drei der bisherigen Mitglieder des Präsidums haben signalisiert, dass sie erneut kandidieren wollen: Ramona Pisal für das Amt der Präsidentin, Margarete Hofmann und Eva Schübel für die beiden Ämter der Vizepräsidentin. Ihre Kandidatur als Schatzmeisterin, Kommissionsvorsitzende und Beisitzerinnen haben angekündigt:

\section{Schatzmeisterin (Präsidium)}

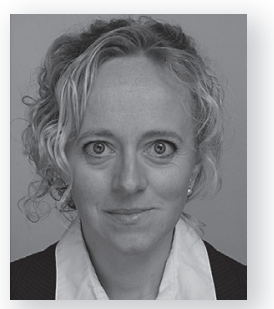

Dr. Karin E. M. Kopp, LL.M. Berkeley, geb. 1975, verheiratet, sieben Kinder, Banklehre in München 1991-1993, Abitur an der BOS Wirtschaft in München 1995, Studium in Bayreuth und Berkeley, Referendarin am OLG Bamberg mit Stationen in München und New York. Zulassung als Rechtsanwältin RAK Stuttgart 2003, State Bar of California 2005, Verzicht auf die Zulassung wegen Verbeamtung auf Lebenszeit 2013. Promotion und Assistentin (Besoldungsgruppe C1) am Lehrstuhl für öffentliches Recht, Verfassungsrecht und Steuerrecht, Universität Bayreuth. Referentin am Max-PlanckInstitut für Steuerrecht und Öffentliche Finanzen in München. Lehrstuhlvertreterin (halbe C 4 Stelle) anlässlich der Emeritierung von Prof. Dr. Gerrit Frotscher am Interdisziplinären Zentrum für Internationales Finanz- und Steuerwesen der Universität Hamburg (IIFS) / International Tax Institute. Wissenschaftliche Mitarbeiterin am Bundesfinanzhof (BFH, VIII. Senat, zuständig für Kapitaleinkünfte und Einkünfte aus selbständiger Arbeit).

Seit Mai 2012 Regierungsrätin, Einstieg in die 4. Qualifikationsebene bei der bayerischen Finanzverwaltung; Einweisung am Finanzamt Wolfratshausen, FA München (BP, BuStrA); Referat 44 (Auslandsfachprüfung) am Bayerischen Landesamt für Steuern (LfSt); Sachgebietsleiterin am Finanzamt München.

Mitglied im djb seit 2009. Mitglied u.a. in ArbeiterKind.de; deutsche Sektion der International Fiscal Association (IFA), Deutschen Steuerjuristischen Vereinigung (DStJG), DeutschAmerikanischen Juristenverein (DAJV); Alumni-Vereine Bayreuth, Berkeley, MPI.

Als Schatzmeisterin unterstütze ich den Wechsel der Kontoverbindung des djb und werde mich u.a. für die Erschließung von Fördermöglichkeiten einsetzen.

Kommission Arbeits-, Gleichstellungs- und Wirtschaftsrecht

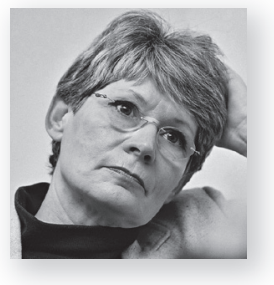

Prof. Dr. Heide Pfarr, geb. 1944. Bis zur Pensionierung Professorin für Arbeitsund Wirtschaftsrecht an der Universität Hamburg. Mannigfache Veröffentlichungen im Arbeitsrecht mit dem Schwerpunkt Gleichstellung der Frauen im Er- werbsleben. 1989 bis 1991 Senatorin in Berlin, danach bis 1994 Staatsministerin für Frauen, Arbeit und Sozialordnung in Hessen, wo ich das (inzwischen in einigen Teilen veränderte) Gleichberechtigungsgesetz für den öffentlichen Dienst entwickelte. 1995 bis 2011 Geschäftsführerin in der gewerkschaftsnahen Hans-Böckler-Stiftung und wissenschaftliche Direktorin des dortigen Wirtschafts- und Sozialwissenschaftlichen Instituts. In dieser Zeit mehrere empirisch gestützte und interdisziplinär angelegte Studien zum Arbeitsrecht. 2002 erarbeitete ich zusammen mit anderen djb-Mitgliedern für die damalige Bundesfrauenministerin einen Entwurf für ein Gleichstellungsgesetz für die Privatwirtschaft, das aber am Kanzler scheiterte. Mitglied im djb seit 1987.

Neben der Begleitung von Gesetzgebungsvorhaben und bedeutenden Verfahren vor den Gerichten möchte ich (nun, da ich Zeit habe) die rechtspolitische Arbeit der Kommission für Arbeits-, Gleichstellungs- und Wirtschaftsrecht voranbringen. Der Gleichstellungsbericht der Sachverständigenkommission, der 2011 dem Bundesministerium für Familie, Senioren, Frauen und Jugend vorgelegt wurde, hat vielfältige Defizite in der Gleichstellung der Frauen im Erwerbsleben aufgezeigt und diese in eindrucksvoller Weise empirisch belegt. Auch konkrete Forderungen wurden gestellt, so im Bereich Minijobs und Wahlarbeitszeiten. Was noch fehlt, sind konkrete Regulierungsvorschläge zur Umsetzung der Forderungen. Dabei sind durchaus komplexe Sachverhalte anzugehen, die nicht nur juristische Kenntnisse, sondern auch Erfahrung, Einblick in betriebliche und gesellschaftliche Praxis und vor allem auch Kreativität erfordern. Genau das, was in der Kommission zusammengekommen ist und zusammen kommen kann.

\section{Kommission Zivil-, Familien- und Erbrecht, Recht anderer Lebensgemeinschaften}

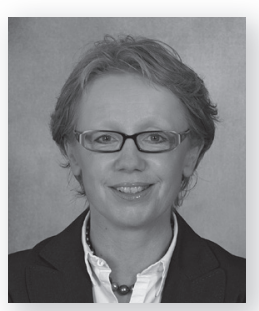

Brigitte Meyer-Wehage, geb. am 10. August 1958 in Ostercappeln (Nds.), verheiratet.

Nach dem Abitur (1977) Studium in Münster (bis 1983) und Referendariat in Oldenburg mit Auslandsstage in Brüssel. Zweites Staatsexamen im August 1986 in Hannover. Danach tätig als Rechtsanwältin in Osnabrück und ab April 1987 in Oldenburg mit Schwerpunkt Versicherungsvertrags- und Baurecht. Wechsel in den richterlichen Dienst des Landes Sachsen-Anhalt zum 1. Juli 1992, u.a. mit Stationen am Landgericht Magdeburg und Oberlandesgericht Naumburg. An beiden Gerichten neben einer Zuweisung zu einer $\mathrm{Zi}$ vilkammer/Zivilsenat auch zuständig für Richterpersonalien (Verwaltung). Von Juli bis Dezember 1997 abgeordnet an die Staatsanwaltschaft II, Berlin, danach Eintritt in den 
Justizdienst des Landes Niedersachen (Richterin am Amtsgericht Delmenhorst mit Zuständigkeit Insolvenzrecht und allgemeines Zivilrecht).

Zum 1. Januar 2003 Abordnung an das Bundesministerium der Justiz (BMJ). Dort Referentin für Familienverfahrensrecht (national und international). Ab Februar 2005 ständige Vertreterin des Direktors beim Amtsgericht Cloppenburg und seit August 2011 Direktorin des Amtsgerichts in Brake. Seit meiner Abordnung an das BMJ bin ich vorrangig im Familienrecht tätig mit Veröffentlichungen in der einschlägigen Fachpresse und Co-Autorin in Vorwerk (Hrsg.), Das Prozessformularbuch.

Gründungsmitglied der Regionalgruppe Oldenburg im djb, Vorsitzende des Landesverbands Niedersachsen im djb seit März 2010. Mitglied der Kommission „Zivil-, Familien- und Erbrecht, Recht anderer Lebensgemeinschaften“ seit 2005, seit November 2012 deren kommissarische Vorsitzende.

Im Familienrecht ist zwar die Anzahl der Gesetzesvorhaben - rückblickend - weniger geworden; Handlungsbedarf besteht aber nach wie vor. Dies gilt zum einen für das Verfahrensrecht, wobei insbesondere über die Einführung der Nichtzulassungsbeschwerde im Familienrecht mit Rücksicht auf den vielbeschriebenen „Gleichlauf der Verfahrensordnungen“ nachzudenken sein wird und zum anderen auch für das materielle Recht. Hier wird z.B. über Alternativen zum gesetzlichen Güterstand und über eine „Neuordnung“ im Adoptionsrecht zu sprechen sein.

\section{Kommission Strafrecht}

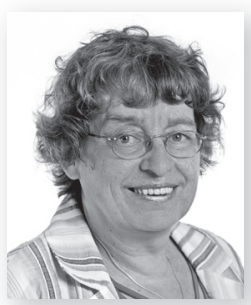

Dagmar Freudenberg, geb. 1952 in Emmern (jetzt: Emmerthal bei Hameln), Studium in Göttingen, verheiratet, zwei Kinder, seit 1978 in der Justiz in Niedersachsen, Staatsanwältin seit 1980 , seit 1981 Bearbeitung von Jugendstrafsachen. Von 1985 bis 1995 Lehrauftrag „Jugendrccht“ am Erziehungswissenschaftlichen Fachbereich der Universität Göttingen. Von 1993 bis 31. März 2009 Bearbeitung des Sonderdezernats „Sexualstraftaten“, parallel dazu seit 2003 auch Bearbeitung des Sonderdezernats „Häusliche Gewalt" bei der Staatsanwaltschaft Göttingen. Ebenfalls seit 2003 auf Bitte des Nds. Ministeriums der Justiz Mitarbeit als Ansprechpartner häusliche Gewalt aus der Staatsanwaltschaft im Praxisbeirat der Landeskoordinierungsstelle häusliche Gewalt beim Landespräventionsrat Niedersachsen.

2006 und 2007 Mitglied in der AG 4 (UAG 1) des Bundesjustizministeriums zur Erarbeitung eines Nationalen Integrationsplans der Bundesregierung und schließlich seit 2002 auf Bitte des Nds. Ministerium der Justiz Mitarbeit in der Stiftung Opferhilfe im Vorstand des Regionalen Opferhilfefonds Göttingen. Ab 2009 zur Hälfte der Arbeitskraft durch Abordnung tätig als Koordinatorin im Projekt häusliche Gewalt im Landespräventionsrat Niedersachsen, gleichzeitig Referentin für häusliche Gewalt und Stalking. Seit 2011 ganz abgeordnet in das Niedersächsische Justizministerium und dort maßgeblich zuständig für die Entwicklung und Durchführung der psychosozialen Prozessbegleitung in Niedersachsen sowie, seit Januar 2013, als Leiterin der Fachstelle Opferschutz im Landespräventionsrat für die Umsetzung der Niedersächsischen Opferschutzkonzeption. Zahlreiche Vorträge, Stellungnahmen und Tagungsveröffentlichungen.

Seit 1994 Mitglied der Strafrechtskommission des djb, von 2001 bis 2009 Vorsitzende der djb-Kommission „Gewalt gegen Frauen und Kinder", seit 2009 Vorsitzende der djb- Kommission „Strafrecht“, dazu seit 2001 für den djb Mitglied der BundLänder-AG „Häusliche Gewalt“ des BMFSFJ und seit 2003 Mitglied der Bund-Länder-AG „Schutz von Kindern und Jugendlichen vor sexueller Gewalt und Ausbeutung “ des BMFSFJ.

Die Fragen der Gleichberechtigung - und insbesondere des Schutzes der zumeist weiblichen Opfer von Gewaltstraftaten - sind mir seit Eintritt in mein Berufsleben, aber auch in der Arbeit des djb besonders wichtig. Ziel meiner Arbeit war und ist die Verwirklichung einer gerechten, gewaltfreien Gesellschaft. Hierfür bedarf es auch und gerade des Engagements in der Strafrechtskommission insbesondere für die Belange der schwachen kindlichen und zumeist weiblichen Opfer. Dazu gehört eine Stärkung der Rechte der Verletzten im Verfahren, zum Beispiel auf (psychosoziale) Opferbegleitung, die Umsetzung der EU-Richtlinie zu Mindeststandards für Opfer von Straftaten 2012/29/EU sowie weiterer europäischer und internationaler Richtlinien und Standards zum Schutz der Opfer von Straftaten ebenso wie der Fortentwicklung des Gewaltschutzes in Recht (GewSchG) und Rechtsalltag. Das schließt die Notwendigkeit der Sensibilisierung, Fortbildung und Ausbildung aller im Bereich der Umsetzung des Rechts tätigen Professionellen, insbesondere in der Justiz, aber auch im Bereich der Anwaltschaft und verschiedener Verbände und Institutionen ein, die durch Vernetzung, Information und Initiativen gewährleistet werden kann und soll. Die Wahrnehmung der Opferrechte im Rahmen des gesetzlich verankerten Deals oder - perspektivisch - eines Ausgleichs im weitesten Sinne (Restorative Justice) bleibt auf der Agenda. An diesen konkretisierten Zielen möchte ich ebenso arbeiten wie an der Verwirklichung der gleichberechtigten Gesellschaft und dafür die Möglichkeiten des Strafrechts und - insbesondere - des Strafprozessrechts einschließlich der Prüfung von Gesetzesreformen nutzen und verbessern.

\section{Kommission Recht der sozialen Sicherung, Familienlastenausgleich}

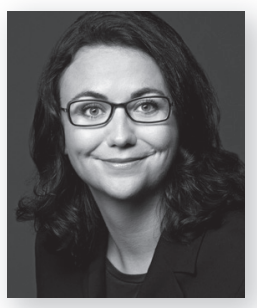

Dr. Maria Wersig, geboren am 28.09.1978 in Weimar, verheiratet. Studium an der Freien Universität Berlin, Promotion zur Dr. phil. 2013 am Fachbereich Erziehungs- und Sozialwissenschaften der Universität Hildesheim (Promotionsthema: Der lange Schatten der Hausfrauenehe. Zur Reformresistenz des Ehegattensplittings). Ich bin seit 2001 Mitglied im Deutschen Juristinnenbund und seit 2009 Mitglied der Kommission Recht der sozialen Sicherung, Familien- 
lastenausgleich. Die Betrachtung der Schnittstellen von Sozialrecht, Familienrecht und Steuerrecht aus der Gleichstellungsperspektive gehört seit 2004 zu meinen Arbeitsschwerpunkten, mit zahlreichen Veröffentlichungen in diesem Themenbereich. Ich verfüge über Berufserfahrung als wissenschaftliche Mitarbeiterin und Lehrbeauftragte an verschiedenen Universitäten und in der Politikberatung als Referentin für Familien- und Gleichstellungspolitik im Bundestag. Zuletzt war ich über 2 Jahre wissenschaftliche Mitarbeiterin am Institut für Sozialund Organisationspädagogik der Universität Hildesheim. Seit Juli 2013 arbeite ich als Referentin des Gesamtbetriebsrats von DB Mobility Logistics in Berlin. Weitere Informationen über mich finden Sie auf meiner Internetseite www.mariawersig.de. Für die Kommissionsarbeit der nächsten Jahre stelle ich mir sowohl die Beobachtung und Begleitung der Reformvorhaben der Politik durch Stellungnahmen und Pressearbeit, als auch die konzeptionelle Auseinandersetzung mit vom politischen Tagesgeschäft unabhängigen Themen, die sich die Kommission gemeinsam setzt, vor. Die eigenständige Existenzsicherung von Frauen und Männern, die Vereinbarkeit von Familie und Beruf und der Umbau der sozialen Sicherungssysteme mit tätigkeitsbezogenen, statt ehebezogenen, Sicherungselementen für Menschen, die Fürsorgearbeit leisten, sollte meiner Ansicht nach im Mittelpunkt unserer Bemühungen stehen. Dabei kann die Kommission an der erfolgreichen Arbeit der Vergangenheit anknüpfen. Einen weiteren Schwerpunkt sehe ich in der Auseinandersetzung mit mittelbarer Diskriminierung im Steuer- und Sozialrecht und der möglichst kommissionsübergreifenden Lobbyarbeit für geschlechterdifferenzierte Gesetzesfolgenabschätzungen in Gesetzgebungsprozessen.

\section{Kommission Öffentliches Recht, Völker- und Europarecht}

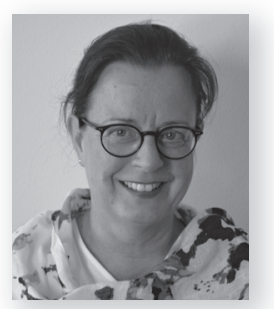

Sabine Overkämping, geb. 1963 in Rhede, Kreis Borken, verheiratet, Studium in Bonn, Schwerpunkt Völker- und Europarecht, seit 1991 als Beamtin des Landes Sachsen-Anhalt in verschiedensten Verwendungen tätig: Koordinatorin für das Votum des Landes im Bundesrat im Ministerium für Bundes- und Europangelegenheiten (1991-1993); dann für das Ministerium der Justiz in der Vertretung des Landes Sachsen-Anhalt beim Bund (1993-1994); im Anschluss Referatsleiterin und stellvertretende Abteilungsleiterin in der Staatskanzlei, zuständig für die Grundsatzangelegenheiten der Frauenpolitik (1994-1998) einschließlich der Novellierung des Frauenfördergesetzes; daraufhin nationale Expertin in der Generaldirektion Beschäftigung und soziale Angelegenheiten der Europäischen Kommission (1997-2000); sodann für das Ministerium für Soziales in der Vertretung des Landes bei der EU (2000-2003); in der Folge zugewiesen an das Verbindungsbüro der ILO (2003-2004); anschließend Referatsleiterin für Europa und Konferenzen im Ministerium für Soziales (2005-2006); daraufhin erneut abgeordnet, und zwar im Rahmen der deutschen Ratspräsidentschaft an das Bundesministerium für Arbeit und Soziales (2006-2009) und derzeit Referatsleiterin für origi- näres Europarecht, Rechtshilfe, Landeskontaktstelle des Europäischen Justiziellen Netzwerkes (EJN) für Zivil- und Handelssachen, materielles Arbeitsrecht und Tarifrecht im Ministerium für Justiz und Gleichstellung (seit 2009); seit 2005 europarechtliche Lehraufträge bei der Otto-von-Guericke-Universität Magdeburg, davor diverse Lehrverpflichtungen im Rahmen der Fort- und Weiterbildung in Sachsen-Anhalt et ailleurs.

Mein djb-Werdegang: Mitte der 90er-Jahre habe ich mit engagierten Kolleginnen in Sachsen-Anhalt den Landesverband Sachsen-Anhalt und die Regionalgruppe Magdeburg gegründet. Lange Jahre war ich Regionalgruppenvorsitzende und habe die Regionalgruppe nach 2001 oft auch im Regionalgruppenbeirat vertreten. Zeitweise war ich Delegierte für UNIFEM (heute: Deutsches Komitee für UN Women), ewla (Europäische Juristinnenvereinigung) und die EBD (Europäische Bewegung Deutschland). Ende der 90er bin ich Mitglied der Kommission Europa geworden und habe in der Folge bis zur Umstrukturierung der Kommissionen und Arbeitsstäbe 2001 den Vorsitz der Kommission Europa übernommen, anschließend war ich Leiterin der Fachgruppe Europa in der Kommission „Öffentliches Recht, Völker- und Europarecht“ und habe diese auch im Bundesvorstand wiederholt vertreten. Das Aufgabenspektrum der Kommission ist mir also vertraut. Vielleicht können wir künftig das Projekt „Aktionärinnen fordern Gleichberechtigung“ auf die europäische Ebene heben. Auch die Hinweise, dass es eine Überarbeitung der europäischen Verträge geben könnte, sind ein Aufgabenfeld. Sicher ist die Nachfolge des Fahrplans für die Gleichstellung auf europäischer Ebene ein Arbeitsfeld sowie die Nachfolge des Stockholmer Programms. Im Öffentlichen Recht gibt es diverse Baustellen. Wir sollten uns verstärkt für die Nachzeichnungspflicht einsetzen und auch eine erleichterte Rückkehr zur Vollzeit nach familienbedingter Teilzeit könnte ein Anliegen sein. Bei den „Migrantinnen“ greife ich hier heraus, dass wir uns für die Abschaffung der Optionspflicht einsetzen sollten.

\section{Beisitzerinnen}

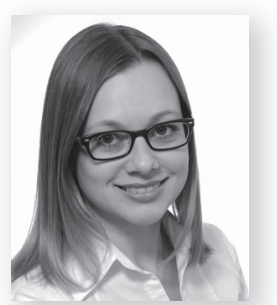

Leonie Babst, Studentin, geboren am 24. April 1991 in Lörrach. Nachdem ich 2010 das Abitur erworben hatte, lebte ich ein Jahr in Französisch-Polynesien, auf Tahiti. Dort studierte ich an der Universität Französisch-Polynesiens französische Literaturwissenschaften. Gleichzeitig war ich als Au-pair-Mädchen in einer Familie tätig. Da ich ein großes Interesse an Sprache und an einem grenzüberschreitenden Dialog habe, entschied ich mich 2011 an der Universität des Saarlandes und am Centre Juridique Franco-Allemand zu studieren. Es handelt sich dabei um einen zweisprachigen binationalen Studiengang. Dies bedeutet, dass ich sowohl ein Studium der Rechtswissenschaften absolviere, als auch eine Licence de droit im französischen Recht anstrebe, welche von der Université de Lorraine vergeben wird. Voraussichtlich werde ich 2014 die Licence erhalten. Besonders reizvoll an dem Doppelstudium ist für mich auch die Aussicht auf einen europäisch oder international orientierten Beruf. 
In der Staatsanwaltschaft Saarbrücken habe ich bereits im Sonderdezernat Häusliche Gewalt ein Praktikum absolviert. Seitdem beschäftigte ich mich verstärkt mit Themen der Gleichberechtigung und des Opferschutzes bei Gewaltstraftaten.

Am djb schätze ich insbesondere, dass junge Studentinnen und Berufseinsteigerinnen von Juristinnen mit Berufserfahrung Unterstützung und Perspektiven erhalten. Um diesen Prozess aktiv zu unterstützen, möchte ich als Beisitzerin im Bundesvorstand kandidieren.

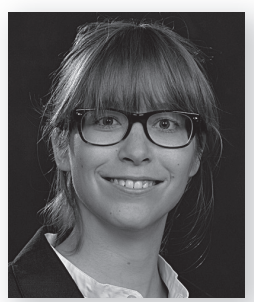

Denise Cordes, Hauptamtliche Leiterin von Justitia Mentoring, dem Frauenförderprogramm an der Rechtswissenschaftlichen Fakultät der AlbertLudwigs-Universität Freiburg i.Br. und Autorin im sechsköpfigen Team einer täglichen juristischen Presseschau bei LegalTribuneOnline.

Geb. 1983 in Bochum, Studium der Rechtswissenschaften mit europarechtlichem Wahlfach an der Ruhr-Universität Bochum, Hilfskraft am Lehrstuhl für Europa- und Völkerrecht (Prof. Dr. Adelheid Puttler, LL.M.). Teilnahme (2006) und anschließende Leitung (2007/ 2008) des Bochumer Philip C. Jessup International Law Moot Court CompetitionTeams. Erstes Staatsexamen 2008. Bis 2012 wissenschaftliche
Mitarbeiterin am Institut für Staatswissenschaft und Rechtsphilosophie, Universität Freiburg; zuvor am Lehrstuhl für Öffentliches Recht, Rechtssoziologie und Rechtsphilosophie an der Universität Bochum (jeweils Prof. Dr. Ralf Poscher). Dissertationsprojekt im Verfassungs- und Völkerrecht zum strafrechtlichen Verbot der Völkermordleugnung. Die weiteren Interessenschwerpunkte liegen im allgemeinen Staatsund Verwaltungsrecht, der feministischen Rechtswissenschaft und der Rechtsphilosophie (Strafrecht).

In Freiburg im Bereich der universitären Selbstverwaltung als Mitglied der Studienkommission und des Fakultätsrates der Rechtswissenschaftlichen Fakultät seit 2010 bzw. 2012 tätig. djb-Mitglied seit 2013. Ehrenamtliches Mitglied im Leitungsteam von Justitia Mentoring und Mentorin im Programm seit 2010 .

Ein besonderes Anliegen wäre mir bei den jungen Juristinnen eine verstärkte Zusammenarbeit mit den Rechtswissenschaftlichen Fakultäten. Mittels der Einführungs- und Orientierungsveranstaltungen kann der djb bei Studentinnen stärker hervortreten. Gerade jungen Frauen stehen dem Konzept der „Frauenförderung“ oft skeptisch gegenüber; gleichzeitig ist der Kontakt mit weiblichen Vorbildern in diesem Rahmen - insbesondere auch mit Blick auf die Vorstellung unterschiedlichster Berufsbilder - und das Netzwerken unter Frauen eine wichtige Erfahrung.

\section{Sitzung des Regionalgruppenbeirates vom 26. bis 28. April 2013 in Augsburg}

\section{Daniela Leukert-Fischer \\ Rechtsanwältin, Vorstand Regionalgruppenbeirat, Vorstand RG Karlsruhe, Bensheim}

Der Regionalgruppenbeirat (RGB) des djb tagt einmal jährlich mit dem Ziel des Informations- und Erfahrungsaustausches zwischen den Regionalgruppen und Landesverbänden. In diesem Jahr kamen vom 26. bis 28. April insgesamt 22 Delegierte aus ganz Deutschland in Augsburg zusammen.

Nachdem das Treffen am Freitagabend mit einem ersten informellen Abendessen begonnen hatte, kamen die Teilnehmerinnen am Samstagmorgen im Ratskeller des Augsburger Rathauses zu ihrer Sitzung zusammen.

Die Präsidentin des djb, Ramona Pisal, berichtete über die Arbeit des djb auf Bundesebene und insbesondere über ihre Tätigkeit im letzten Jahr und die Besuche der verschiedensten Veranstaltungen wie auch die Teilnahme an diversen Hauptversammlungen im Rahmen unseres Projekts „Aktionärinnen fordern Gleichberechtigung “. Auch unsere Vizepräsidentin, Eva Schübel, war zur Sitzung angereist, um die Teilnehmerinnen zu begrüßen und einen Zwischenstand zur erfolgreichen
Aktion „Rote Roben“ sowie zum Netzwerk der Gleichstellungsbeauftragten zu geben.

Bundesgeschäftsführerin Anke Gimbal gab einen Überblick über die geplanten Veranstaltungen, insbesondere den nächsten Bundeskongress in Leipzig vom 26. bis 29. September

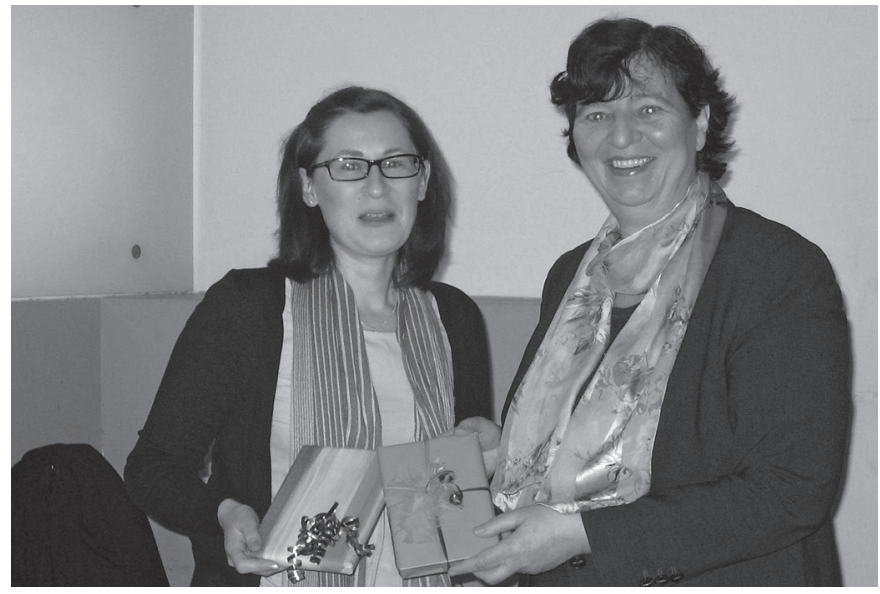

A Verabschiedung von Rechtsanwältin und vereidigte Buchprüferin Birgit Kersten (rechts), Brake, nach sechs Jahren Amtszeit als RGB-Vorstandsmitglied, mit Rechtsanwältin Daniela Leukert-Fischer, Bensheim. Bild: Christina Lorenz. 